\title{
CW Silver Ion Laser with Electron Beam Excitation
}

\author{
B. WERNSMAN, T. PRABHURAM, K. LEWIS, F. GONZALEZ, M. VILlaGRAN, ANd J. J. ROCCA
}

\begin{abstract}
A CW laser power of $140 \mathrm{~mW}$ was obtained in the 840.39 $\mathrm{nm}$ transition of $\mathrm{Ag}$ II by electron beam excitation. This electron beam excited metal vapor ion laser is capable of operating using metals with high vaporization temperatures and is of interest for generation of $\mathrm{CW}$ coherent radiation in the $220-260 \mathrm{~nm}$ spectral region.
\end{abstract}

$P^{2}$ REVIOUSLY we reported laser action in ionic and atomic transitions excited by charge transfer reactions [1] and three-body electron-ion recombination [2] in electron beam generated plasmas. $\mathrm{CW}$ laser action was obtained in more than 50 infrared and visible lines using $\mathrm{dc}$ electron beam excitation. In all cases, the active medium was a mixture of helium with another gas or a metal vapor with a relatively low melting point $(<900 \mathrm{~K})$. In the case of the blue lines of $\mathrm{Zn} \mathrm{II,} \mathrm{an} \mathrm{order} \mathrm{of} \mathrm{magnitude} \mathrm{increase}$ in the laser output power and efficiency was obtained with respect to previously used hollow cathode discharges [1]. The electron beam charge transfer excitation scheme also has the potential of producing $\mathrm{CW}$ laser light with higher output powers than presently obtainable in the 220-260 $\mathrm{nm}$ spectral region. In this case, the active medium must contain $\mathrm{Ag}, \mathrm{Cu}$, or $\mathrm{Au}$ vapor [3]-[6]. The required metal vapor concentrations are on the order of $10^{15}$ atoms $\mathrm{cm}^{-3}$, and the associated vaporization temperatures are between 1400 and $2100 \mathrm{~K}$. The optimum metal vapor concentration depends on the plasma density, temperature, and radius. This optimum vapor concentration is the minimum metal atom density required to make charge transfer the dominant loss channel for the noble gas ions over diffusion to the walls and electron-ion recombination.

Molecular donors have been used to produce concentrations of these atoms [7], [8], but their use is unsuitable in ultraviolet lasers because of the unacceptable high losses caused by absorption of the laser radiation by molecular transitions. Here we discuss an electron beam laser capable of operating with metal vapors requiring a high vaporization temperature, and we report its $\mathrm{CW}$ operation in the $840.39 \mathrm{~nm}\left(6 p^{3} F_{4}^{\circ}-6 s^{3} D_{3}\right) \mathrm{Ag}$ II transition. In a $\mathrm{He}-\mathrm{Ag}$ plasma, the $6 p^{3} F_{4}^{\circ}$ level is excited by charge transfer collisions from the ground state helium ions in a similar manner as the $5 d^{1} S_{0}$ and $5 d^{3} D_{2} \mathrm{Ag}$ II levels from which the 224.34 and $227.76 \mathrm{~nm}$ laser lines originate, re-

Manuscript received January 11, 1988; revised March 7, 1988. This work was supported by AFOSR Grant 87-0290 and a National Science Foundation Presidential Young Investigators Award. The work of M. Villagran was supported by a fellowship from the Organization of American States. The work of J. J. Rocca was supported by an NSF Presidential Young Investigator Award.

The authors are with the Department of Electrical Engineering, Colorado State University, Fort Collins, CO 80523.

IEEE Log Number 8821732. spectively. The metal vapor concentrations required for laser oscillation in both cases are also similar. The results obtained in the infrared are consequently relevant to future attempts at using an electron beam to excite the ultraviolet lines.

A simplified diagram of the laser is illustrated in Fig. 1. A molybdenum tube $90 \mathrm{~cm}$ in length and with a $0.9 \mathrm{~cm}$ inside diameter contains the noble gas-metal vapor plasma. The plasma tube is surrounded by a heating coil made of a tantalum tube cut in a helical fashion to provide an adequate resistance for ohmic heating: $0.57 \Omega$ at 2000 $\mathrm{K}$. Boron nitride disks support the plasma tube from both ends, allowing for free expansion. The tantalum ribbon heater structure is considerably more rugged than heaters based on a refractory metal wire wound around a ceramic tube used in other types of lasers [9], [10]. We have heated the structure from room temperature to $1500 \mathrm{~K}$ in less than 20 min repeatedly without failure. More important, the entire plasma tube-heater assembly is very compact as required to be inserted inside the bore of a $2.2 \mathrm{~cm} \mathrm{I.D.}$ electromagnet which provides a magnetic field of up to $3.5 \mathrm{kG}$. In longitudinally excited $\mathrm{CW}$ electron beam lasers, the magnetic field is vital to guide and confine the electron beam, allowing for efficient deposition of the electron beam power into the plasma. The inner stainless steel tube of the electromagnet is part of the vacuum system. Its inner wall is carefully polished to enhance the reflectivity of the radiation emitted by the heater and increase its efficiency. Since the entire heater-plasma tube structure is in a low pressure environment, heat losses by convection are also reduced. To obtain a temperature of $1500 \mathrm{~K}$ in the plasma tube, $4.25 \mathrm{~kW}$ of heater power is required when the electron guns are not operated. When electron beam plasma excitation takes place, the heater power required is lower because the majority of the electron beam power deposited into the plasma is transferred to the walls of the plasma tube by line radiation and particle collisions.

A helium flow is maintained through the electron gun chambers as indicated in Fig. 1. The pressure in both chambers is maintained equal to avoid significant migration of metal vapor from the plasma tube volume. Due to the geometry of the plasma tube (length/diameter = 100 ), the metal vapor is well contained. We have operated the laser $\mathrm{CW}$ nearly $100 \mathrm{~h}$ without significant loss of metal.

Plasma excitation is achieved using two glow discharge electron guns capable of producing a total electron beam current of $1 \mathrm{~A}$ at an energy of several $\mathrm{keV}$. The electron guns contained sintered molybdenum-magnesium oxide 


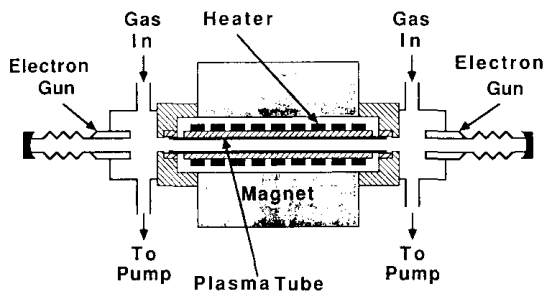

Fig. 1. Schematic representation of the electron beam excited metal vapor laser capable of operating with metals having a high vaporization temperature.

cathodes that allow the generation of a high de electron beam current in oxygen-free atmospheres [11].

Hereafter, we discuss results obtained using this device to generate $\mathrm{CW}$ infrared laser radiation in the $840.39 \mathrm{~nm}$ $\left(6 p^{3} F_{4}^{\circ}-6 s^{3} D_{3}\right)$ line of $\mathrm{Ag}$ II. When the electron beam current is $300 \mathrm{~mA}$ and no external heat is provided, helium lines dominate the spectrum. As the heater current is incremented, the plasma turns green due to the emission from the 520.91, 546.55, and 547.16 nm Ag I lines. At this condition, when a power of $2.0 \mathrm{~kW}(24 \mathrm{~V}, 84 \mathrm{~A})$ was dissipated by the heater coil, the plasma tube temperature reached approximately $1425 \mathrm{~K}$, corresponding to a silver vapor pressure of $75 \mathrm{mtorr}$. CW laser oscillation in the $840.39 \mathrm{~nm}$ line of $\mathrm{Ag}$ II by charge transfer excitation from ground state helium ions created by electron impact was readily achieved. The electron beam current threshold for oscillation was only $45 \mathrm{~mA}$ when a 7.3 percent transmissive output coupler was used, indicating that the small-signal gain at this current is on the order of 0.1 $\mathrm{cm}^{-1}$. A comparison of the current threshold of oscillation of the infrared and ultraviolet $\mathrm{Ag}$ II and $\mathrm{Cu}$ II lines in hollow cathode discharges, which are also negative glow plasmas, shows that the thresholds for the ultraviolet lines are nearly an order of magnitude higher than the infrared thesholds [12]. A similar current increase could in principle be expected for oscillation in the case of an electron beam excited ultraviolet laser. However, such a direct comparison of the threshold currents might not apply since in the sputtering hollow cathodes, a minimum current is always necessary to produce the metal vapor concentration, and consequently, the current requirements for sputtering and excitation are not separated. Therefore, it is possible that the ratio of threshold current for oscillation in both spectral regions in the electron beam plasma might be larger than indicated by a simple comparison in sputtering hollow cathodes.

The variation of the laser output power of the 840.39 $\mathrm{nm} \mathrm{Ag}$ II line as a function of current and voltage is shown in Fig. 2. A linear increase with electron beam current was observed up to a value of $350 \mathrm{~mA}$, after which the laser output power continues to increase sublinearly up to the maximum current investigated. Observation of the $840.39 \mathrm{~nm}$ spontaneous emission shows a similar sublinear increase of the laser upper level population with electron beam current and voltage, as shown in Fig. 3. The spontaneous emission of the He I 501.6 and 586.5 $\mathrm{nm}$ lines which are excited by electron impact and by cas-

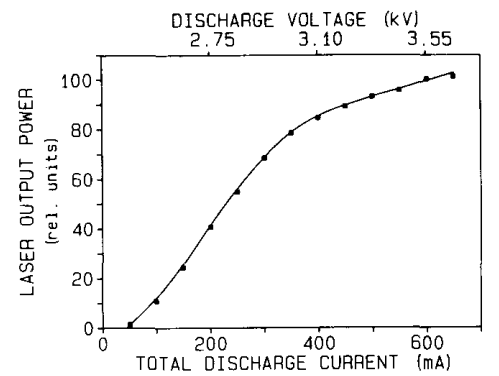

Fig. 2. CW laser output power of the $\mathrm{Ag}$ II $840.39 \mathrm{~nm}$ transition as a function of discharge current and voltage. The magnetic field was 2.8 $\mathrm{kG}$. The helium pressure was 2.2 torr, and the input power on the heater coil was $3.7 \mathrm{~kW}$.

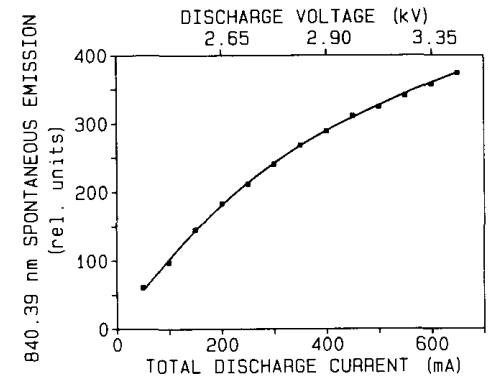

Fig. 3. Spontaneous emission from the $840.39 \mathrm{~nm}$ spectral line as a function of discharge current and voltage.

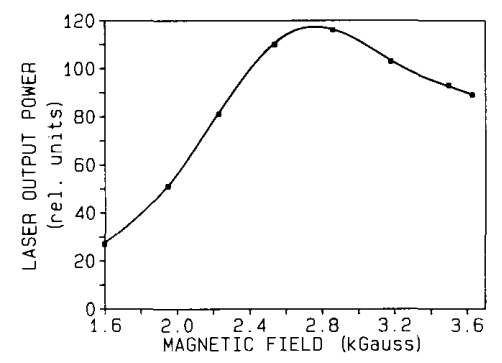

Fig 4 CW laser output power of the Ag II $840.39 \mathrm{~nm}$ transition as a function of magnetic field strength. The discharge current was $600 \mathrm{~mA}$, and the helium pressure was 2.2 torr. The heater coil input power was $3.67 \mathrm{~kW}$.

cade following the recombination of the He ions also have the same dependence. This effect is probably due to the lower ionization cross section of $\mathrm{He}$ at higher electron beam energies which decreases the electron beam power deposition efficiency into the gas target.

The effect of the externally applied axial magnetic field on the laser output power is shown in Fig. 4. As discussed above, the axial magnetic field helps to guide the electron beam into the plasma and minimizes the scattering of beam electrons to the plasma tube. However, the magnetic field also confines the cold secondary electrons. A larger density of cool thermal electrons $\left(T_{e}=0.1 \mathrm{eV}\right)$ can increase the loss of helium ground state ions by threebody electron-ion recombination and also depopulate the laser upper level by superelastic collisions. Consequently, a magnetic field strength increase above $2.8 \mathrm{kG}$ 
results in a decrease in the laser output power. The maximum laser output power was measured to be $0.14 \mathrm{~W}$ and was obtained at $825 \mathrm{~mA}$ of discharge current using a nonoptimized optical cavity with 7.3 percent transmissivity made with $2 \mathrm{~m}$ radius of curvature mirrors. This resonator configuration allowed us to make use of only approximately 10 percent of the total plasma volume. Optimization of the optical cavity to allow a better overlap of the mode volume with the volume of the electron beam created plasma should result in substantially larger laser output powers, in the vicinity of $1 \mathrm{~W}$.

In summary, we obtained a laser output power of 140 $\mathrm{mW}$ in the $840.39 \mathrm{~nm}$ transition of $\mathrm{Ag}$ II. CW laser oscillation from metals having a high vaporization temperature can be obtained from the device described in this paper. This electron beam excited laser should prove particularly useful in generating CW UV ion laser radiation in the $220-260 \mathrm{~nm}$ spectral region.

\section{ACKNOWLEDGMENT}

The authors want to thank M. Ratner, W. Akers, and R. Kiesel for their assistance and acknowledge previous colaborations with J. D. Meyer and G. J. Collins. We also thank W. Silfvast for encouraging discussions.

\section{REFERENCES}

[1] J. J. Rocca, J. D. Meyer, and G. J. Collins, " $1-W$ CW Zn ion laser," Appl. Phys. Lett., vol. 43, pp. 37-39, 1983.

[2] J. J. Rocca, H. L. Mancini, and B. Wernsman, "Cd recombination laser in a plasma generated by an electron beam," IEEE J. Quantum Electron., vol. QE-22, pp. 509-512, 1986.

[3] J. R. McNeil, W. L. Johnson, G. J. Collins, and K. B. Persson, "Ultraviolet laser action in He-Ag and Ne-Ag mixtures," Appl. Phys. Lett., vol. 29, pp. 172-174, 1976.

[4] J. R. McNeil, G. J. Collins, K. B. Persson, and D. L. Franzen, " CW laser oscillation in Cu II," Appl. Phys. Lett., vol. 27, pp. 595-598, 1975.
[5] K. Jain and S. A. Newton, "Operating characteristics of UV and IR hollow-cathode silver, gold and copper ion lasers," Appl. Phys. B, vol. 26 , pp. 43-48, 1981.

[6] J. J. Rocca and G. J. Collins, "Ultraviolet ion lasers," Optoelectron. Instrum. Data Processing, vol. 1, pp. 1-16, 1984.

[7] J. J. Rocca, J. D. Meyer, B. G. Philstrom, and G. J. Collins, “CW laser action in atomic florine,"' IEEE J. Quantum Electron., vol. QE20, pp. 625-628, 1984.

[8] J. A. Piper and D. F. Neely, "CW laser oscillation on transitions of $\mathrm{Cu}^{+}$in He-Cu halide gas discharges," Appl. Phys. Lett., vol. 33, pp. 621-623, 1978.

[9] M. Piltch and G. Gould, "High temperature alumina discharge tube for pulsed metal vapor lasers," Rev. Sci. Instrum., vol. 37, pp. 925927, 1966.

[10] J. J. Kim and J. F. Convey, "High-temperature insulation for the copper vapor laser,"' Rev. Sci. Instrum., vol. 53, pp. 1623-1624, 1982.

[11] J. J. Rocca, J. D. Meyer, M. R. Farrell, and G. J. Collins, “Glowdischarge-created electron beams: Cathode materials, electron gun designs, and technological applications," J. Appl. Phys., vol. 56, pp. 790-797, 1984.

[12] J. R. McNeil, "New sputtered metal vapor laser systems," dissertation, Colorado State Univ., Fort Collins, 1977.

B. Wernsman, photograph and biography not available at the time of publication.

T. Prabhuram, photograph and biography not available at the time of publication.

K. Lewis, photograph and biography not available at the time of publication.

F. Gonzalez, photograph and biography not available at the time of publication.

M. Villagran, photograph and biography not available at the time of publication.

J. J. Rocca, photograph and biography not available at the time of publication. 